\title{
Association of lifestyle behaviours with self-esteem through health-related quality of life in Spanish adolescents
}

\author{
Emily Knox $^{1}$ • Jose Joaquin Muros ${ }^{1,2}$
}

Received: 10 October 2016 / Revised: 19 February 2017 / Accepted: 23 February 2017 / Published online: 6 March 2017

(C) The Author(s) 2017. This article is published with open access at Springerlink.com

\begin{abstract}
The present research examined the association of Mediterranean diet adherence and physical activity with self-esteem through five components of healthrelated quality of life. Data were collected from 456 adolescents attending one of five schools in Granada, Spain using a cluster-randomised design. Participants completed questionnaires on Mediterranean diet adherence, physical activity, self-esteem and health-related quality of life (HRQoL). Models were constructed to identify associations between Mediterranean diet adherence and physical activity on self-esteem. Mediational analysis using bootstrapped confidence intervals examined possible mediation by five components of HRQoL. Mediterranean diet adherence and physical activity engagement were associated with four components of HRQoL: more positive physical wellbeing, psychological wellbeing, family relationships and autonomy support and perceptions of the school environment. Both lifestyle behaviours were positively associated with self-esteem. Both relationships were mediated through positive psychological wellbeing and perceptions of the school environment. Physical wellbeing
\end{abstract}

Communicated by Peter de Winter

Emily Knox

Emily.knox@nottingham.ac.uk

Jose Joaquin Muros

jjmuros@ugr.es

1 School of Health Sciences, University of Nottingham, Queens Medical Centre, Nottingham, UK

2 Department of Nutrition and Food Science, University of Granada, Granada, Spain was also a mediator of the relationship between physical activity and self-esteem.

Conclusion: Interventions promoting Mediterranean diets or physical activity to adolescents may facilitate improvements in self-esteem in addition to wider health benefits previously identified. Approaches within such interventions targeting improvements in physical wellbeing, psychological wellbeing and positive perceptions of the school environment may improve their efficacy.

What is Known:

- It is known that engagement in lifestyle behaviours such as physical activity is positively linked with psychological health.

- Whilst its consumption is declining, the Mediterranean diet is nutritionally recommended and remains popular in parts of Greece, Southern Italy and Spain. Research into Mediterranean diet adherence and psychological health is lacking.

What is New:

- The present research furthers this knowledge by examining potential mechanisms through which two lifestyle behaviours (physical activity and following a Mediterranean diet) may be associated with self-esteem.

- Implications for the promotion of positive mental health in young people. Mediterranean diet and physical activity were positively associated with self-efficacy via positive psychological self-concept and perceptions of the school environment. These novel findings can contribute to the development of more efficacious interventions targeting positive self-esteem in young people.

Keywords Mediterranean diet · Physical activity · Self-esteem $\cdot$ Health-related quality of life $\cdot$ Adolescents
Abbreviations
BMI Body mass index
HRQoL Health-related quality of life
PAQ-C Physical activity questionnaire for older children 


\section{Introduction}

Adolescence is the period in human growth and development that occurs after childhood and before adulthood, between the ages of 10 and 19 years $[21,22]$. It can be an especially challenging period of the life course as rapid cognitive, physical, psychological and emotional changes take place which impinge on health and wellbeing [3]. It is estimated that around 20\% of Spanish individuals will encounter some form of mental health disorder at some point during their adolescence [17]. For these reasons the mental health of young people is a national priority in Spain [17] and across Europe [38]. Low-self-esteem is associated with lower academic achievement and anxiety, depression and eating disorders [1]. On the other hand, high self-esteem has been associated with good mental health and developing and protecting self-esteem has been advocated as a key approach in prevention and mental health promotion [15]. The school offers a potential setting for intervention as aspects such as school peers have been found to have a strong impact on self-esteem during adolescence [32].

Engaging in healthy lifestyle behaviours, such as physical activity or following a healthy diet, is associated with positive self-esteem and mental health. For instance, physical activity has been associated with positive self-esteem in adolescents $[2,19,20,28]$. Adhering to a Mediterranean diet, characterised by high consumption of olive oil, fruits, vegetables, whole grains, moderate to high consumption of fish, moderate consumption of milk and dairy products and low consumption of meat and meat products [35], is suggested to also relate to more positive mental health outcomes [11]. Despite this, only around $22.8 \%$ of Spanish adolescents (aged 11-17 years old) meet physical activity guidelines [39]. In addition, adherence to a Mediterranean diet is also low [30]. In the last 40 years, noticeable modifications to the dietary habits of adolescents have been observed in the Mediterranean countries, resulting in an increase in the consumption of processed food, refined sugar, saturated fats and cholesterol [29]. These two behaviours (physical activity and MD adherence) therefore offer a potential opportunity for targeting improved mental health of adolescents.

Improved understanding of the mechanisms through which physical activity or Mediterranean diet adherence can improve self-esteem would facilitate the development of more effective interventions. In a recent study by Breslin and colleagues [4], positive associations were identified between physical activity and aspects of health-related quality of life in 9- to 11-year-old children. The authors called for greater consideration of the specific relationships between wellbeing and physical activity when conducting interventions with children. The present research aims to identify the channels through which selfesteem is most likely to be enhanced by physical activity. We will explore whether physical activity and adherence to a Mediterranean diet are associated with self-esteem through five different components of health-related wellbeing (physical, psychological, family relationships and autonomy, peer relationships and social and the school environment). The findings will inform the development of more effective interventions within similar adolescent populations.

\section{Methods}

\section{Subjects}

Participants were recruited from their schools to participate in this cross-sectional research. Between 2014 and 2015, there were 20,929 adolescents enrolled at schools across Granada. The study involved 456 adolescents aged between 11 and 14 years, of which 235 were girls and 221 boys. Demographic characteristics of the study sample are provided in Table 1. Data were collected between March and May in 2014. Power analysis suggested that the study required a minimum sample of 378 adolescent to achieve sufficient power with a $95 \%$ confidence interval $(\alpha 0.05 ; \beta 0.2)$. Five of the 55 public schools in the city centre of Granada (Spain) were randomly selected to participate in this research. All participating schools were in a medium-high socioeconomic area based on information contained in the educational project of the centre or school. All adolescents from the five schools aged between 11 and 14 years $(N=511)$ were invited to take part in this study. The sample was recruited from five schools in Granada (Spain) in a cluster-randomised design. Five hundred and eleven adolescents were selected and invited to take part in this study. Of these, 480 agreed to participate and written informed consent was received from their parent or guardian. Twentyfour adolescents were excluded for failing to complete some element of testing, or because they failed to attend class on their

Table 1 Baseline characteristics of the study sample

\begin{tabular}{ll}
\hline & Sample $(N=456)$ \\
\hline Age (years) & $12.57 \pm 1.17$ \\
Gender $(\%$ male) & $51.5 \%$ \\
BMI $\left(\mathrm{kg} / \mathrm{m}^{2}\right)$ & $19.75 \pm 3.85$ \\
Physical activity (score) & $2.92 \pm 0.64$ \\
Mediterranean diet adherence (score) & $7.87 \pm 2.08$ \\
Self-esteem (score) & $33.13 \pm 5.37$ \\
Physical wellbeing (score) & $52.79 \pm 12.11$ \\
Psychological wellbeing (score) & $52.70 \pm 11.08$ \\
Family relationships and autonomy support (score) & $50.57 \pm 9.60$ \\
Social relationships and peer pressure (score) & $54.86 \pm 10.16$ \\
School environment (score) & $54.34 \pm 10.04$ \\
\hline
\end{tabular}

Data shown as mean \pm SD. Mediterranean diet adherence: $\geq 8$, good; 4-7, average; $\leq 3$, poor

$B M I$ body mass index 
testing day. Both the adolescent and their parents or guardians were informed of the objectives and methods of the study and told that they could withdraw at any time. Participants were instructed on how to fill out the questionnaires and how to conduct the tests. All tests were conducted during participants' physical education lesson in school time. No incentives were provided to adolescents or parents. A research assistant was also on hand to provide guidance on the completion of questionnaires and conduct physical testing. Ethical approval was granted by the ethics committee of the University of Granada. Ethical principles of the Declaration of Helsinki for medical research were adhered to.

\section{Health-related quality of life}

To assess health-related quality of life (HRQoL), we used the KIDSCREEN-27 questionnaire [34]. This internationally validated instrument [25] has been applied in populations of healthy and chronically ill children and adolescents aged from 8 to 18 years. The KIDSREEN-27 consists of 27 items relating to five components (physical wellbeing, psychological wellbeing, autonomy and relationship with parents, social support and peers and school environment). Internal consistency of the subscales was between 0.81 and 0.84 , and the test-retest reliability of the subscales ranged from 0.61 to 0.74 [20]. Higher scores indicate higher HRQoL.

\section{Anthropometric measurement}

Height and weight were measured following the protocols established by the International Society for the Advancement of Kinanthropometry [31] using a stadiometer (GPM, Seritex, Inc., Carlstadt, $\mathrm{NJ} ; \pm 1 \mathrm{~mm}$ accuracy) and an electronic scale (model 707, Seca Corporation, Columbia, MD; \pm 50 g accuracy); body mass index (BMI) was calculated as weight divided by height squared $\left(\mathrm{kg} / \mathrm{m}^{2}\right)$.

\section{Physical activity}

Physical activity levels were evaluated using the Physical Activity Questionnaire for Older Children (PAQ-C). The questionnaire provides a general measure of physical activity for 8- to 20-year-olds. The PAQ-C is a self-administrated questionnaire consisting of nine items rated on a five-point scale. A higher score indicates more active children. Respondents are asked to recall the frequency and type of physical activity they have engaged in on each of the 7 days prior to completing the questionnaire. Validation studies have found the PAQ-C to be highly reliable [27].

\section{Adherence to the Mediterranean diet}

Adherence to the Mediterranean diet was assessed using the Evaluation of the Mediterranean Diet Quality Index (KIDMED) [30] which was created to estimate adherence to the Mediterranean diet in children and young adults. The test comprises 16 dichotomous items (yes/no) of which 12 items describe behaviours consistent with the Mediterranean diet, e.g. "Do you use olive oil at home?" and four items describe behaviours inconsistent with the Mediterranean diet, e.g. "Do you consume sweets and candy several times every day?". Affirmative answers to Mediterranean diet consistent and inconsistent behaviours were scored +1 and -1 respectively, giving a maximum possible score of 12 .

\section{Self-esteem}

Self-esteem was evaluated using the Rosenberg self-esteem scale [26]. This self-report questionnaire consists of 10 items rated on a four-point Likert scale, ranging from 1 (strongly disagree) to 4 (strongly agree). Five items are positively worded (e.g. "On the whole, I am satisfied with myself"), and five are negatively worded ("Sometimes I feel really useless"). A minimum score of 10 points and a maximum score of 40 points are possible, with higher scores indicating higher self-esteem. The scale was translated and validated with Spanish students [16], showing satisfactory internal consistency $(0.85$ to 0.88$)$ and test-retest reliability $(0.84)$.

\section{Statistical analysis}

Path analysis using SPSS 22.0 was employed to evaluate whether the five components of HRQoL explained (i.e. mediated) the relationships between the independent variables (physical activity and adherence to the Mediterranean diet) and self-esteem. One model was created for physical activity and one model was created for adherence to Mediterranean diet. Both models were adjusted to control for BMI and gender. Bootstrapping was also applied to the models in order to improve statistical rigour. Bootstrapping analyses were conducted following the method of Preacher and Hayes [23] for estimating indirect effects in simple mediation models. To establish mediation using this method, four paths should be created and analysed. The first path is the simple effect of the independent variable on self-esteem (path $a$ ). The second path is the effect of the independent variable on the proposed mediator (the five components of HRQoL [path $b]$ ). The third path is the effect of the proposed mediator on self-esteem (path $c$ ) The final path (path $c^{\prime}$ ) is the direct effect of the independent variable on selfesteem, controlling for paths $a$ and $b$. Bootstrapped estimates of paths $a$ (independent variable $\rightarrow$ mediator), $b$ (mediator $\rightarrow$ self-esteem), $c^{\prime}$ (direct effect of independent variable $\rightarrow$ selfesteem) and $c$ paths (total effect of independent variable $\rightarrow$ self- 
esteem) were performed. As advised by Hayes [10], a causal steps approach was not used and the indirect effect was evaluated even when path $c$ was non-significant. Bootstrapped estimates of path $c^{\prime}$ were performed to test the model by which the predictor has no effect in the criterion when the mediator is controlled (i.e. moderation). Finally, the true indirect effect for the mediation models was tested through bootstrapped estimates of the product of paths $a$ and $b\left(a^{*} b\right)$. Statistical significance for each path tested was established when zero did not lie between the $95 \%$ bootstrapped confidence interval, with 1000 bootstrap resamples.

\section{Results}

\section{Mediterranean diet and self-esteem}

The first indirect effect model used adherence to the Mediterranean diet as the independent variable and the five components of subjective wellbeing (physical, psychological, family relationships and autonomy support, social relationships and peer pressure and school environment) as potential mediators. Results from this set of bootstrapped estimates are provided in Fig. 1 and described below.

Analyses suggested that Mediterranean diet was directly associated with self-esteem, i.e. when any confounding influence of the potential mediators was not considered (path $c$; $\beta=0.37, \mathrm{SE}=0.15, p<0.05$ ). Path $c^{\prime}$, which examined the relationship of Mediterranean diet through the mediating variables, was non-significant ( $\beta=0.12, \mathrm{SE}=0.13, p=0.35$ ), suggesting that the influence of following a Mediterranean diet on self-esteem was not mediated by all five components of subjective wellbeing. Values for each component were therefore scrutinised separately. Mediterranean diet was not associated with social relationships and peer pressure (path $a ; \beta=0.41, \mathrm{SE}=0.25, p=0.11$ ). Physical self-concept and family relationships and autonomy support were then examined as mediator variables. The relationship between Mediterranean diet and each of these variables was significant (paths $a ; \beta=1.19, \mathrm{SE}=0.29, p<0.001 ; \beta=0.50, \mathrm{SE}=0.23$, $p<0.05)$. However, the relationship between these variables and self-esteem was not significant (path $b$ ), and so these variables were not explored further.

Mediterranean diet was significantly associated with psychological self-concept (path $a ; \beta=0.76, \mathrm{SE}=0.28, p<0.01$ ). Further, psychological self-concept was associated with selfesteem (path $b ; \beta=0.20, \mathrm{SE}=0.0 .03, p<0.001$ ). Thus, the indirect effects were then tested by examining bootstrapped estimates of path $a^{*} b$. Examination of confidence intervals suggested that psychological self-concept mediated the association between Mediterranean diet and self-esteem, since zero was not included ( $\beta=0.15, \mathrm{SE}=0.06, \mathrm{CI}=0.04$ to 0.30 ). Examination of the standardised effect $(\beta=0.06, \mathrm{SE}=0.02$, $\mathrm{SE}=0.02$ to 0.11$)$ and ratio of indirect to total effect $(\beta=1.25$, $\mathrm{SE}=22.38)$ produced the same conclusions.

Mediterranean diet was significantly associated with perceptions of the school environment (path $a ; \beta=1.08$, $\mathrm{SE}=0.23, p<0.001$ ). Further, perception of the school environment was associated with self-esteem (path $b ; \beta=0.06$, $\mathrm{SE}=0.03, p<0.05$ ). The indirect effects were also then tested. Examination of bootstrapped confidence intervals of path $a^{*} b$ suggested that the school environment mediated the association between Mediterranean diet and self-esteem, since zero was not included $(\beta=0.06, \mathrm{SE}=0.03, \mathrm{CI}=0.01$ to 0.15 ). Examination of the standardised effect $(\beta=0.02, \mathrm{SE}=0.01$, $\mathrm{CI}=0.01$ to 0.05$)$ and ratio of indirect to total effect $(\beta=0.52$, $\mathrm{SE}=10.73)$ produced the same conclusions.

\section{Physical activity and self-esteem}

The second indirect effect model used engagement with physical activity as the independent variable and the five
Fig. 1 Mediational analysis of the influence of adherence to the Mediterranean diet (MD) on selfesteem through the five components of wellbeing. The solid bold line shows the direct effect of MD on self-esteem (path $c^{\prime}$ ). Solid light lines show significant effects of the predictor (MD) on the mediator variables (HRQoL) (path $a$ ) and the significant effects of the mediator variables on self-esteem (path $c$ ). Dashed lines show nonsignificant effects

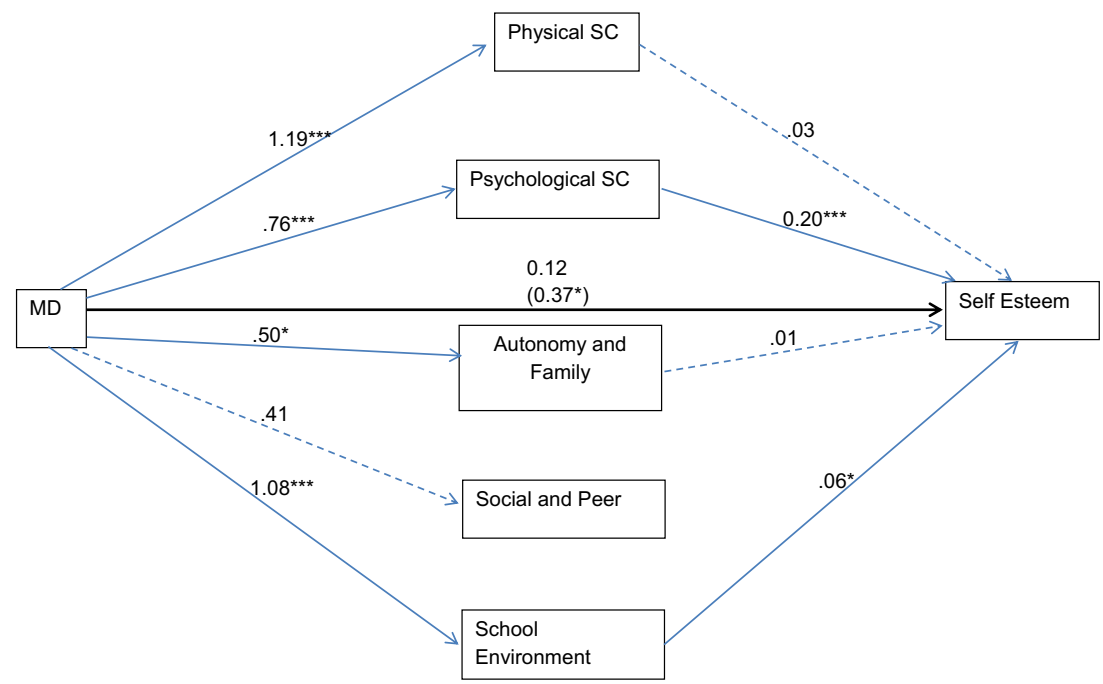


components of subjective wellbeing (physical, psychological, family relationships and autonomy support, social relationships and peer pressure and school environment) as potential mediators. Results from this set of bootstrapped estimates are provided in Fig. 2 and described below.

Analyses suggested that physical activity was not directly associated with self-esteem, when any influence of the potential mediating variables was not considered (path $c ; \beta=0.64$, $\mathrm{SE}=0.49, p=0.19$ ). Path $c^{\prime}$ (association of physical activity on self-esteem, when the potential mediating variables were considered) was significant $(\beta=-1.03, \mathrm{SE}=0.48, p<0.05)$, suggesting that physical activity did exhibit a relationship with self-esteem through the five components of subjective wellbeing. These mediation effects were further investigated. Physical activity was related with family relationships and autonomy support and social relationships and peer pressure (path $a ; \beta=3.95, \mathrm{SE}=0.77, p<0.001 ; \beta=3.27, \mathrm{SE}=0.75$, $p<0.001)$. However, none of these components of HRQoL were related to self-esteem (path $b$ ), and so these variables were not explored further.

Physical activity was related with physical self-concept (path $a)(\beta=9.73, \mathrm{SE}=0.86, p<0.001)$. Further, physical self-concept was associated with self-esteem (path $b$; $\beta=0.06, \mathrm{SE}=0.03, p<0.05)$. Bootstrapped confidence intervals of the indirect effects were then examined. Path $a^{*} b(\beta=0.61, \mathrm{SE}=0.28, \mathrm{CI}=0.06$ to 1.14$)$ was significant as confidence intervals did not include zero, suggesting that physical self-concept mediated the relationship between physical activity and self-esteem. Examination of the standardised effect $(\beta=0.07, \mathrm{SE}=0.03, \mathrm{CI}=0.01$ to $0.13)$ and ratio of indirect to total effect $(\beta=-0.59$, $\mathrm{SE}=13.72$ ) produced the same conclusions.

Physical activity was associated with psychological selfconcept (path $a ; \beta=4.37, \mathrm{SE}=0.82, p<0.001$ ). Further, psychological self-concept was associated with self-esteem (path $b ; \beta=0.20, \mathrm{SE}=0.03, p<0.001)$. Path $a^{*} b(\beta=0.87$, $\mathrm{SE}=0.21, \mathrm{CI}=0.54$ to 1.38 ) was significant suggesting that psychological self-concept also mediated the relationship between physical activity and self-esteem. Examination of the standardised effect $(\beta=0.10, \mathrm{SE}=0.02, \mathrm{CI}=0.06$ to 0.15$)$ and ratio of indirect to total effect $(\beta=-0.85, \mathrm{SE}=54.51)$ produced the same conclusions.

Physical activity was associated with perceptions of the school environment (path $a ; \beta=3.27, \mathrm{SE}=0.75$, $p<0.001)$. Further, the school environment was associated with self-esteem (path $b ; \beta=0.06, \mathrm{SE}=0.03, p<0.05$ ). Path $a^{*} b(\beta=0.21, \mathrm{SE}=0.10, \mathrm{CI}=0.04$ to 0.45$)$ was also significant, suggesting that the school environment mediated the relationship between physical activity and self-esteem. Examination of the standardised effect $(\beta=0.02, \mathrm{SE}=0.01$, $\mathrm{CI}=0.01$ to 0.05$)$ and ratio of indirect to total effect $(\beta=-0.20, \mathrm{SE}=10.06)$ produced the same conclusions.

\section{Discussion}

Results from the present study suggest that adolescents who follow a Mediterranean diet tend to hold more positive perceptions of their physical wellbeing, psychological wellbeing, autonomy support and family relationships and of their school environment, regardless of their BMI or gender. Further, these adolescents also exhibit more positive self-esteem, and this appears to be partly attributable to the influence of following a Mediterranean diet on their psychological wellbeing and perceived school environment.

A recent study [37] identified a number of psychopathological benefits to be associated with Mediterranean diet adherence in Spanish school-aged children. This included reduced risk of depression or suffering from an eating disorder and low anxiety. The present study is the first to demonstrate
Fig. 2 Mediational analysis of the influence of physical activity engagement on self-esteem through the five components of wellbeing

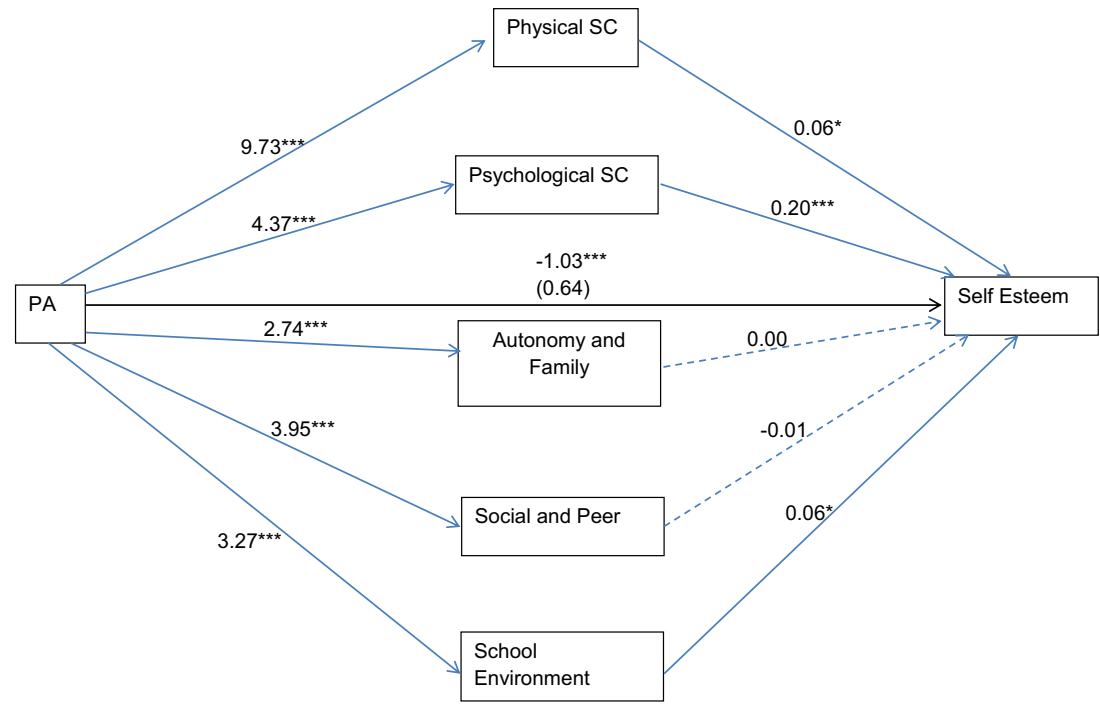


the association of Mediterranean diet adherence with selfesteem through improved psychological wellbeing in adolescents. Georgiadis et al. [9] conducted cluster analyses according to self-esteem theory on a Greek sample of dieters. Worryingly, less than $30 \%$ demonstrated an adaptive psychological profile characterised by high self-esteem and less controlling diets. The Mediterranean diet has demonstrated vast benefits to health $[6,35,36]$. Further, adolescents adhering to a Mediterranean diet in our sample and throughout Spain as part of their regular lifestyle may be protected from the negative psychological aspects of restrictive dieting [9]. Crichton and colleagues [6] have also uncovered that a Mediterranean style diet is related with improved psychological functioning in Australian adults even when adherence was not high. Eating foods consistent with the Mediterranean diet could therefore be important across the lifespan. Further research is required to uncover the mechanism through which the Mediterranean diet might exert this influence.

The present study also suggests that positive perceptions of the school environment positively impacts self-esteem in active adolescents who adhere to a Mediterranean diet. Previous research has linked Mediterranean diet adherence to better academic performance of Mediterranean children [8,36], and academic performance has been linked to self-esteem [24]. It is possible that the adolescents in the present sample had higher academic attainment which led to them more positively perceiving their school environment. As academic attainment of the present sample was not measured, further research including observational studies may be useful to illuminate the reasons for these more positive perceptions of the school environment.

The second part of this research revealed that physically active adolescents also tended to respond more positively to all five components of wellbeing. The associations with physical activity were much stronger than those for Mediterranean diet adherence. Further, physical activity was positively associated with self-esteem through positive associations with physical wellbeing, psychological wellbeing and perceptions of the school environment.

The relationship between physical activity and physical and psychological wellbeing has been explored in numerous different populations [14, 18]. Strauss and colleagues [33] have also identified the importance of physical activity to the development of self-esteem in 10- to 16-yearolds. This is the first study to identify these constructs as mediators of self-esteem in Spanish adolescents. Moreno et al. [18] reported that physical activity positively influenced self-esteem and physical wellbeing specifically in a sample of 2332 students aged 9-23 years. These authors also identified gender and age differences. Other research has suggested that overweight adolescents may especially benefit from engaging in physical activity in terms of selfesteem [28]. The present research identified mediating effects of physical wellbeing on self-esteem. Many adolescents experience physical changes which can lower their self-esteem [13], and it is possible that physically active adolescents possess a healthy body image of themselves simply because they are active [12]. The present findings indicate that physical activity could be especially critical at this time to maintain positive physical wellbeing. Further, psychological wellbeing may be especially important for maintaining high levels of motivation to be active [7]. Interventions to increase physical activity levels of adolescents should, therefore, seek to raise adolescent's self-referenced perceptions of physical and psychological wellbeing to improve their self-esteem.

Positive perceptions of the school environment also mediated the relationship between physical activity and self-esteem. Previous research has suggested that engagement in physical activity mediates perceptions of school-related stress with more active children reporting less felt stress [5]. The more positive perceptions of the school environment of active adolescents may be at least partly explained by their experience of less stress. It is also possible that these adolescents perceived more opportunities to practice physical activity at school. This reinforces suggestions that schools should be utilised as the setting of physical activity interventions.

\section{Limitations}

Conclusions from the present research should be interpreted in light of a number of limitations. The research design was cross-sectional and so inferences around causality cannot be made. Further, self-report methods were employed which introduces possible measurement error. However, as both the IPAQ-C and the KIDMED have previously demonstrated high validity and reliability in this population, we believe that this should have little impact on the conclusions made. Further, interactions between physical activity behaviour and dietary habits could have a further influence on self-esteem which was not addressed in the present study. Future research could aim to do so. It would also be interesting to compare the present population with those from other areas of Spain. Given the findings relating to the perception of the school environment, it could be particularly interesting to examine rural areas, where the school environment is likely to be very different to that found in a city. Despite these limitations, this is the first study, to our knowledge, to analyse associations between Mediterranean diet adherence and physical activity on self-esteem through HRQoL in adolescents.

\section{Conclusions}

The present study suggests that adolescents who follow a Mediterranean diet or who engage in more physical 
activity exhibit higher self-esteem. The original contribution of this research is the finding that both of these behaviours may relate to self-esteem through positive psychological self-concept and perceptions of the school environment. This has important implications for parents, teachers, youth workers, policy-makers and other professionals with a responsibility to protect the psychological health of young people. One approach might be to encourage schools to be the settings of behavioural interventions, either through provision of opportunities to engage in the behaviours or provision of information promoting them. Other approaches could be to provide educational sessions within Mediterranean diet or physical activity interventions which encourage a positive body image. However, it is not only the schools who must shoulder responsibility. Policy-makers should reinvigorate efforts to educate parents and their children about the benefits of physical activity and the Mediterranean diet and direct resources to aid provision in schools. Where possible, youth workers should seek to offer opportunities to experience both, i.e. through cooking workshops or taster classes, whilst paediatricians should be able to both educate patients and signpost to community-based opportunities. Further studies are required to identify how healthy lifestyle approaches can have the most positive effect on self-esteem via psychological wellbeing, physical wellbeing and perceptions of the school environment.

Authors' contributions Emily Knox: Participated in the design of the study and contribute to developing of the research protocol, performed data analysis, drafted the initial manuscript and approved the final manuscript as submitted.

Jose Joaquin Muros: Participated in the design of the study and contribute to developing of the research protocol, collected data, supervised drafting of the manuscript and approval the final manuscript as submitted.

Compliance with ethical standards Ethical approval was granted by the ethics committee of the University of Granada.

Funding No external funding was secured for this study.

Conflict of interest The authors declare that they have no conflict of interest.

Financial disclosure The authors have no financial relationships relevant to this article disclose.

Ethical approval All procedures performed in studies involving human participants were in accordance with the ethical standards of the institutional and/or national research committee and with the 1964 Helsinki Declaration and its later amendments or comparable ethical standards.

Informed consent Informed consent was obtained from all individual participants included in the study.
Open Access This article is distributed under the terms of the Creative Commons Attribution 4.0 International License (http:// creativecommons.org/licenses/by/4.0/), which permits unrestricted use, distribution, and reproduction in any medium, provided you give appropriate credit to the original author(s) and the source, provide a link to the Creative Commons license, and indicate if changes were made.

\section{References}

1. Bartels M, Cacioppo JT, Van Beijsterveldt TC, Boomsma DI (2013) Exploring the association between well-being and psychopathology in adolescents. Behav Genet 43(3):177-190. doi:10.1007/s10519. 013-9589-7

2. Biddle SJ, Asare M (2011) Physical activity and mental health in children and adolescents: a review of reviews. Br J Sports Med 45(11):886-895. doi:10.1136/bjsports-2011-090185 \nbjsports2011-090185

3. Braddick F, Carral V, Jenkins R, Jane-Llopis E (2009) Child and adolescent mental health in Europe: infrastructures, policy and programmes. European Communities, Luxembourg

4. Breslin G, Gossrau-breen D, Mccay N, Gilmore G, Macdonald L, Hanna D (2012) Physical activity, gender, weight status, and wellbeing in 9- to 11-year-old children: a cross-sectional survey. J Phy Act Health 9:394-401

5. Brown J, Siegel J (1988) Exercise as a buffer of life stress: a prospective study of adolescent health. Health Psychol 7:341-355

6. Crichton GE, Bryan J, Hodgson JM, Murphy KJ (2013) Mediterranean diet adherence and self-reported psychological functioning in an Australian sample. Appetite 70:53-59. doi:10. 1016/j.appet.2013.06.088

7. Davison KK, Werder JL, Trost SG, Baker BL, Birch LL (2007) Why are early maturing girls less active? Links between pubertal development, psychological well-being, and physical activity among girls at ages 11 and 13. Soc Sci Med 64(12):2391-2404. doi:10.1016/j.socscimed.2007.02.033

8. Esteban-Cornejo I, Izquierdo-Gomez R, Gomez-Martinez S, Padilla-Moledo C, Castro-Pinero J, Marcos A, Veiga OL (2016) Adherence to the Mediterranean diet and academic performance in youth: the UP\&DOWN study. Eur J Nutr 55(3):1133-1140. doi:10.1007/s00394-015-0927-9

9. Georgiadis M, Biddle S, Stavrou N (2006) Motivation for weightloss diets: a clustering, longitudinal field study using self-esteem and self-determination theory perspectives. Health Educ J 65(1): 53-72. doi:10.1177/0017896906066067

10. Hayes AF (2009) Beyond Baron and Kenny: statistical mediation analysis in the new millennium. Commun Monogr 76(4):408-420

11. Jacka FN, Kremer PJ, Berk M, de Silva-Sanigorski AM, Moodie M, Leslie ER et al (2011) A prospective study of diet quality and mental health in adolescents. PLoS One 6(9):1-7. doi:10.1371/ journal.pone. 0024805

12. Kirkcaldy BD, Shephard RJ, Siefen RG (2002) The relationship between physical activity and self-image and problem behaviour among adolescents. Soc Psychiatry Psychiatr Epidemiol 37(11): 544-550. doi:10.1007/s00127-002-0554-7

13. Kort-Butler LA, Hagewen KJ (2011) School-based extracurricular activity involvement and adolescent self-esteem: a growth-curve analysis. J Youth Adolesc 40(5):568-581. doi:10.1007/s10964010-9551-4

14. Lu A, Hong X, Yu Y, Ling H, Tian H, Yu Z, Chang L (2015) Perceived physical appearance and life satisfaction: a moderated mediation model of self-esteem and life experience of deaf and 
hearing adolescents. J Adolesc 39:1-9. doi:10.1016/j.adolescence. 2014.11.005

15. Mann M, Hosman CM, Schaalma HP, De Vries NK (2004) Selfesteem in a broad-spectrum approach for mental health promotion. Health Educ Res 19(4):357-372. doi:10.1093/her/cyg041

16. Martín-Albo J, Núñez JL, Navarro JG, Grijalvo F (2007) The Rosenberg self-esteem scale: translation and validation in university students. Span J Psychol 10(2):458-467

17. Ministerio de Sanidad, \& Servicios Sociales e Igualdad (2014) Difusión de la Estrategia en Salud Mental del Sistema Nacional de Salud y formación a profesionales. Ministerio de Sanidad y Consumo, Madrid

18. Moreno JA, Cervello E, Moreno R (2008) The importance of physical-sport practice and gender in physical self-concept from 9 up to 23 years. Int J Clin Health Psychol 8(1):171-183

19. Ortega FB, Ruiz JR, Castillo MJ, Sjöström M (2008) Physical fitness in childhood and adolescence: a powerful marker of health. Int J Obes 32(1):1-11. doi:10.1038/sj.ijo.0803774

20. Parfitt G, Eston RG (2005) The relationship between children's habitual activity level and psychological well-being. Acta Paediatr 94(12):1791-1797. doi:10.1080/08035250500268266

21. Patton G, Sawyer S, Santelli JS, Ross DA, Afifi R, Allen NB et al (2016) Our future: a Lancet commission on adolescent health and wellbeing. Lancet 387:2423-2478

22. Patton GC, Viner R (2007) Pubertal transitions in health. Lancet 369(9567):1130-1139. doi:10.1016/S0140-6736(07)60366-3

23. Preacher KJ, Hayes AF (2004) SPSS and SAS procedures for estimating indirect effects in simple mediation models. Behav Res Methods Instrum Comput 36(4):717-731. doi:10.3758/BF03206553

24. Pullmann H, Allik J (2008) Relations of academic and general selfesteem to school achievement. Pers Individ Dif 45(6):559-564. doi: 10.1016/j.paid.2008.06.017

25. Ravens-Sieberer U, Gosch A, Rajmil L, Erhart M, Bruil J, Duer W et al (2005) KIDSCREEN-52 quality-of-life measure for children and adolescents. Expert Rev Pharmacoecon Outcomes Res 5(3): 353-364. doi:10.1586/14737167.5.3.353

26. Rosenberg M (1965) Society and the adolescent self-image. University Press, Princeton

27. Saint-Maurice PF, Welk GJ, Beyler NK, Bartee RT, Heelan KA (2014) Calibration of self-report tools for physical activity research: the Physical Activity Questionnaire (PAQ). BMC Public Health 14(461):1-9. doi:10.1186/1471-2458-14-461

28. Schmalz DL, Deane GD, Birch LL, Davison KK (2007) A longitudinal assessment of the links between physical activity and self- esteem in early adolescent non-Hispanic females. J Adolesc Health 41(6):559-565. doi:10.1016/j.jadohealth.2007.07.001

29. Serra-Majem L, García-Closas R, Ribas L, Pérez-Rodrigo C, Aranceta J (2001) Food patterns of Spanish schoolchildren and adolescents: the enKid study. Public Health Nutr 4:1433-1438. doi:10.1079/PHN2001234

30. Serra-Majem L, Ribas L, Ngo J, Ortega RM, Garcia A, PerezRodrigo C, Aranceta J (2004) Food, youth and the Mediterranean diet in Spain. Development of KIDMED, Mediterranean diet quality index in children and adolescents. Public Health Nutr 7(7):931935. doi:10.1079/PHN2004556

31. Stewart A, Marfell-Jones M, Olds T, de Ridder H (2011) International standards for anthropometric assessment. ISAK, Lower Hutt

32. Strange J, Neuenschwander N, Dauer A (2005) Self-esteem in females throughout childhood and adolescence. URJHS 4:1-17

33. Strauss R, Rodzilsky D, Burack G, Colin M (2001) Psychosocial correlates of physical activity in healthy children. Arch Pediatr Adolesc Med 155(8):897-902

34. The KIDSCREEN Group Europe (2006) The KIDSCREEN questionnaires - quality of life questionnaires for children and adolescents. Pabst Science Publishers, Lengerich

35. Trichopoulou A, Costacou T, Bamia C, Trichopoulos D (2003) Adherence to a Mediterranean diet and survival in a Greek population. N Engl J Med 348(26):2599-2608. doi:10.1056/ NEJMoa025039\r348/26/2599

36. Vassiloudis I, Yiannakouris N, Panagiotakos DB, Apostolopoulos $\mathrm{K}$, Costarelli V (2014) Academic performance in relation to adherence to the Mediterranean diet and energy balance behaviors in Greek primary schoolchildren. J Nut Educ Behav 46(3):164-170. doi:10.1016/j.jneb.2013.11.001

37. Voltas N, Arija V, Aparicio E, Canals J (2016) Longitudinal study of psychopathological, anthropometric and sociodemographic factors related to the level of Mediterranean diet adherence in a community sample of Spanish adolescents. Public Health Nutr 20:1-11. doi:10.1017/S1368980015003560

38. World Health Organization (2015) The European mental health action plan 2013-2020. Geneva. Retrieved from http://www.euro. who.int/ data/assets/pdf file/0020/280604/WHO-EuropeMental-Health-Acion-Plan-2013-2020.pdf

39. World Health Organization (2013) Spain physical activity factsheet. Geneva. Retrieved from http://www.mom.gov.sg/ workplace-safety-health/worker-workplace-surveillance/Pages/ default.aspx 